\title{
AC 2011-1921: POSTER SESSION FOR TENURE TRACK FACULTY
}

Donald P. Visco, Tennessee Technological University

Jason M. Keith, Michigan Technological University

Jason Keith is an Associate Professor of Chemical Engineering at Michigan Technological University.

Dr. Jeffrey A Nason, Oregon State University

Roger C. Lo, Department of Chemical Engineering, California State University, Long Beach

Roger C. Lo is an Assistant Professor of Chemical Engineering at California State University, Long Beach. He received his PhD from Texas A\&M University in May 2008. Roger teaches undergraduate and graduate required courses (fluids, math, and transport phenomena) and also numerical analysis using Excel and MATLAB for chemical engineering calculations. Roger's research interest focuses on microfluidics and its applications to solving chemical and biological problems, such as fuel cells, microreactors, and high-throughput chemical/biological assays.

Dr. James P Abulencia, Manhattan College

Sergio Mendez, California State University, Long Beach

(C)American Society for Engineering Education, 2011 


\author{
Poster Session for Tenure Track Faculty \\ Organized by: \\ Donald P. Visco, Jr. \\ Department of Chemical and Biomolecular Engineering \\ Associate Dean for Undergraduate Studies \\ The University of Akron
}

\author{
And \\ Jason M. Keith \\ Department of Chemical Engineering \\ Michigan Technological University
}

\begin{abstract}
ASEE Annual Meeting. The goal of the session is to allow tenure-track faculty or instructors to share their research efforts and their pedagogical interests with their peers at other institutions. The posters will have about half of the material related to research related pursuits with the rest containing information on teaching efforts/interests. A hopeful outcome of the session is that new faculty/instructors be given the opportunity to discuss collaborations with faculty at another university to aid the goals of their research and/or teaching.
\end{abstract}




\section{Jeffrey A. Nason, Ph.D. \\ School of Chemical Biological and Environmental Engineering Oregon State University}

\section{Research Interests}

My research interests are in the areas of aquatic chemistry and physical/chemical treatment processes, with a focus on particle dynamics in natural and engineered systems. Current research is focused on understanding the fate and transport of engineered nanomaterials in the environment. The rapid development of novel nanomaterials and their incorporation into consumer products has not been paralleled with an equal effort investigating the possible environmental implications. Increasingly, the properties of engineered nanoparticles (ENPs) are tailored for specific applications through the use of organic capping agents. The properties imparted by the capping agents are also likely to influence the transport, toxicity and fate of these materials in the environment. In addition to the purposeful tailoring of ENPs, it is well established that natural organic matter (NOM), which is ubiquitous in surface water, will interact with natural colloids and ENPs, influencing their environmental behavior. The overarching objective of our current work is to develop an improved understanding of the roles of synthetic capping agents and NOM in controlling the homogeneous and heterogeneous aggregation of ENPs in aquatic environmental systems. The poster will discuss recent results examining the interactions between NOM and gold nanoparticles with different capping agents.

\section{Teaching/Education Interests}

My teaching responsibilities include undergraduate courses in Material Balances and Air Pollution Control. I also teach graduate courses in Aquatic Chemistry and Physical/Chemical Treatment Processes. In all of these courses, I strive to incorporate active learning techniques and peer-instruction, along with appropriate uses of technology in the classroom. The Material Balances and Stoichiometry course is a large interdisciplinary course that is required of chemical, biological and environmental engineers in our program. In this course, I utilize a Web-based Interactive Science and Engineering (WISE) learning tool developed here at OSU. The WISE learning tool allows the instructor to pose questions to the class that probe for conceptual understanding and supports a variety of student response types including: multiple choice answers, multiple choice with short answer follow-up, numerical answers, short answers, and Likert-scale surveys. The tool provides formative assessment of student learning, allows for immediate feedback by the instructor, provides rich opportunities for peer-instruction, and facilitates education research.

At present we are exploring the extent to which student performance on WISE activities, perception of WISE as a useful teaching and learning tool, and participation using WISE in the Material Balances course correlates with student approaches to learning as determined by the Colorado Learning Attitudes about Science Survey (CLASS) and the Revised two-factor Study Process Questionnaire (R-SPQ-2F). In promoting conceptual understanding, we hypothesize that the WISE activities will appeal to students who take a deep approach to learning. However, realizing that students' perceptions of the WISE tool will also be influenced by factors including the active learning environment and the immediate feedback it promotes, these aspects of student perception will be evaluated in an additional survey of student opinions at the end of the course. This work is part of a longitudinal study which seeks to determine the extent to which active learning approaches can change students' approaches to learning. 


\title{
Microfluidics@ the Beach \\ Roger C. Lo \\ Department of Chemical Engineering \\ California State University, Long Beach
}

\begin{abstract}
Research Interests
Microfluidics involves the study of the behavior of fluids at microscale, fluid manipulations, and the design of the devices that can effectively perform such manipulations. It has been widely applied to the miniaturization of analytical methods and chemical and biological processes because of its many advantages, such as significant reduction in analysis time, much lower sample and reagent consumption (in the nanoliter range or less), and enhanced system performance and functionality by integrating different components onto microfluidic devices. These applications are usually called micro total analysis systems ( $\mu \mathrm{TAS}$ ) or lab on a chip (LOC). Our research interest focuses on microfluidics and its application to solving chemical and biological problems, such as fuel cells, microreactors, and high-throughput chemical/biological assays.
\end{abstract}

\section{Teaching / Education Interests}

In addition to microfluidics research, we are also interested in the aspect of microfluidics education, especially when we notice that from the microfluidics technology industry (especially in California) there is a need for chemical engineers with related skills, such as microfluidic chip design, microfabrication, optical imaging, and programming languages for instrument control and data analysis. However, our current curriculum at CSULB does not provide our students training for these skills as an integrated module. To address this, we initiated a course development project for two new elective courses, Introduction to Microfabrication and microfluidics Technology and Microfluidics Technology and Its Applications, along with corresponding hands-on lab sessions. In the first course, the fundamentals of microfluidics, chip design, and microfabrication techniques are introduced in both class lectures and related readings. In the lab sessions, students will actually go to our research laboratory to design and fabricate microfluidic chips using soft lithography. In the second course, the applications of microfluidics technology, e.g., in chemistry, engineering, and biotechnology, are introduced through class lectures and journal paper readings. In the lab session, students will perform experiments on their microfluidic chips, such as DNA electrophoresis, mixing, organic synthesis, and fuel cell reactions, to get familiar with fluid manipulations, proper calibration of detectors, and data analysis. They will also learn how to use programming languages, e.g., MATLAB, and software packages, e.g., ImageJ, to process numerical and image data.

In this project, undergraduate and graduate students are involved in the design of the lab sessions. They help convert the experiments in our ongoing research projects into the ones suitable for teaching by actually performing them and revising the protocol to fit our class needs. Through this project, the students obtain not only the working knowledge of microfluidics technology, but also the communication skills required for effective technical information exchange. 


\title{
Research and Teaching Interests of Professor Sergio Mendez Sergio Mendez \\ Department of Chemical Engineering Department California State University, Long Beach
}

\begin{abstract}
Research Interests
Titanium dioxide (TIO2) is widely used as a photocatalyst. In this work, we employ sol-gel chemistry to synthesize nanostructued TIO2 microspheres. To enhance the photocatalytic activity, we incorporate carbon nanotubes into the microspheres. Furthermore, the exposed surfaces of the TIO2 and carbon nanotubes are chemically modified. The particle size and morphology are characterized with scanning electron microscopy, the nanostructure is determined with transmission electron microscopy and x-ray diffraction, and the chemical composition with FTIR spectroscopy. We demonstrated control over the mesoporosity, crystallinity, morphology and surface chemical composition. To quantify the photocatalytic activity of the TIO2 particles, we measure the photodecomposition of a fluorescent dye under ultraviolet irradiation. A parametric study was performed to find the optimum conditions for maximum photocatalytic activity.
\end{abstract}

\section{Teaching / Education Interests}

In the chemical engineering curriculum, students are taught about the fundamentals of heat and momentum transfer. The teaching process involves classroom lectures and often corresponding undergraduate laboratory experiments. Another tool that can be used to reinforce the concepts introduced in the classroom and practiced in the lab is computer simulation. The benefits of using COMSOL are many: 1) it is designed to model heat, momentum, mass, etc. transfer; 2) ease of learning the software; 3 ) the ability to have either simple or complicated models; 4) quick simulation time; 5) and relative low cost. We have developed two systems that incorporate COMSOL simulations. In one lab, students perform a simple steady-state transient heat conduction experiment. This experimental data can used to estimate the thermal conductivity, $k$, and thermal diffusivity, $a$, of a material in the shape of slab. A COMSOL model can then be constructed with $k$ and $a$ as the inputs to model the transient heat transfer through the material. The students can make a direct comparison between their experimental findings and the COMSOL simulation. In another application, the students can use the Navier-Stokes equations to derive the parabolic velocity profile of liquid flowing through a narrow slit. Now the students can make a direct comparison between a theoretical prediction and a COMSOL simulation. We hope that introducing students to COMSOL will intrigue them to explore the power of the software, especially the built-in "Chemical Engineering" module. 


\section{James Patrick Abulencia \\ Department of Chemical Engineering Department \\ Manhattan College}

\section{Research Interests}

My research interests are under the broad umbrella of bioengineering, and follow three primary trajectories. In the first, we seek sustainable materials and methods for water purification in developed nations. The group has been working with a small community in the Philippines to use natural materials such as coconut derived activated carbon to clean water. In the second, we develop ways to efficiently produce biofuels. Candidate organisms used in these studies include yeast and algae. In the final line of research, we examine the magnitude of bacterial adhesion onto surfaces. Bacteria called Actinomyces Acetocomitans participates in the pathogenesis of juvenile periodontis by strongly binding to teeth. This adhesion force, which has traditionally been measured using atomic force microscopy (AFM), is measured using tribometry techniques.

\section{Education Research}

My research interest broadly addresses the assertion that Chemical Engineering education must be revised to address a new generation of student. This student is information rich, technology savvy, and must be trained to address the consequences of globalization. Thus, the education we received as students may not be the best way to approach this group. To this end, we have

performed research on engineering ethics, the use of social networking, and video learning in an effort to address the new skill set and resources available to our students. 